\title{
Viel Arbeit, wenig Zeit - trotzdem glücklich
}

\author{
Ärzte sind glücklich in ihrem Beruf - eigentlich. Es gibt jedoch immer noch zu \\ viele Wermutstropfen, belegt der „Ärztemonitor": zu viel Bürokratie, zu wenig \\ Zeit für den Patienten. Die Kassen zeigen sich wenig beeindruckt.
}

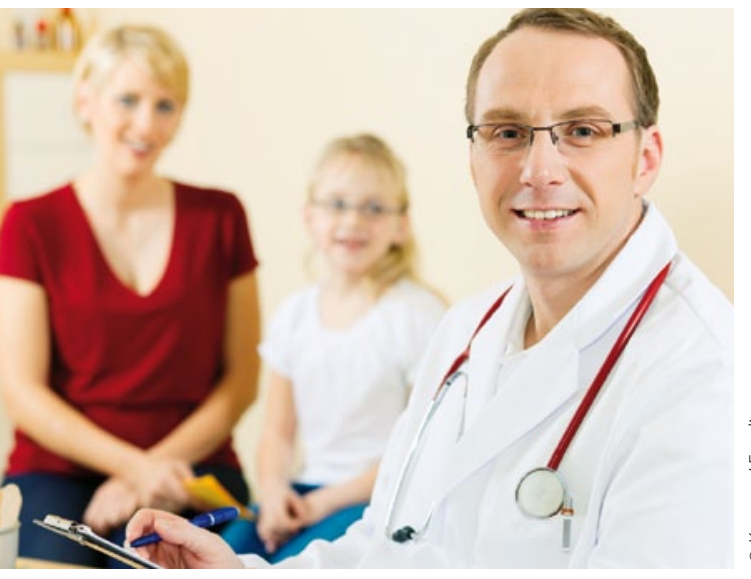

Die meisten Ärzte sind hinreichend zufrieden mit ihrem Beruf.

— Eigentlich könnte alles so schön sein: $72 \%$ der niedergelassenen Ärzte empfinden ihre Arbeit als nützlich und sinnvoll. 53\% stimmen der Aussage „meine Arbeit macht mir Spaß“ voll zu. 59\% würden den Arztberuf wieder ergreifen. Das hat eine Umfrage unter 11000 niedergelassenen Ärzten und Psychotherapeuten ergeben, die die KBV gemeinsam mit dem NAV-Virchow-Bund in Auftrag gegeben hatte. Sogar mit ihrem Einkommen seien die meisten Ärzte einverstanden: 49\% der Ärzte gaben an, sie seien „eher zufrieden“, lediglich $10 \%$ sind „sehr unzufrieden" mit ihrer finanziellen Situation. Der Berufsstand ziehe seinen Selbstwert nicht nur aus materiellen Aspekten, so KBV-Chef Dr. Andreas Köhler.

\section{Hohe Patientenzahlen}

Dennoch: Es gibt Wermutstropfen. 50\% der Befragten gaben an, dass sie am Ende des Tages „,öllig erledigt“ seien. 29\% fühlten sich nach getaner Arbeit sogar "ausgebrannt“. Das betrifft vor allem die Gruppe zwischen 45 und 49 Jahren, die im Schnitt eine hohe Patientenzahl bewältigen. Hausärzte versorgen laut der Studie 53 Patienten am Tag, bei Fachärzten sind es täglich 41 Patienten. Psychotherapeuten, die im Durchschnitt sieben Patienten pro Tag behandeln, sind mit ihrem Beruf zufriedener, sagt Robert Follmer, Studienleiter des "Ärztemonitors“.

\section{Zu viel Büroarbeit}

Im Schnitt arbeiten Haus- und Fachärzte 55 Stunden in der Woche. Nur etwa $60 \%$ der Zeit entfielen dabei auf Sprechstunden. Zu viel Zeit entfiele auf
Bürokratie, die den zweitgrößten Anteil an der Gesamtarbeitszeit ausmacht, so Köhler.

\section{Kritik der Krankenkassen}

Der GKV-Spitzenverband beurteilte den Ärztemonitor kritisch: Ärzte könnten leicht mehr Zeit für ihre Patienten haben, sagte Verbandssprecher Florian Lanz. Der Verband habe 2009 selbst eine Umfrage durchgeführt, nach der niedergelassene Ärzte im Schnitt 28,5 Stunden pro Woche Sprechstunde haben. Rechne man Termine nach Vereinbarung hinzu, kämen Fachärzte auf knapp 32 Stunden. Die große Mehrheit der Hausärzte biete Hausbesuche an und erreiche so durchschnittlich 36 Stunden. „Den zeitlichen Spielraum für mehr Sprechzeiten scheint es auf jeden Fall zu geben“, so Lanz.

SUNNA GIESEK -

\section{DEGAM-Programm für Studenten}

\section{Bereits im Studium zur Allgemeinmedizin kommen}

Um Medizinstudenten möglichst früh für die Allgemeinmedizin zu begeistern, hat die Deutsche Gesellschaft für Allgemeinmedizin und Familienmedizin (DEGAM) eine Nachwuchsakademie gegründet. 12 Studierende - neun Frauen, drei Männer - wurden für das dreijährige Förderprogramm ausgewählt. Anfang Juli trafen sie sich in Frankfurt/ Main zum ersten Klausurwochenende.

Das Studium der Allgemeinmedizin hat an den deutschen Universitäten nicht das beste Image. Viele Studierende fühlen sich isoliert, nicht ernst genommen und mit Vorurteilen konfrontiert. „Hier in der Gruppe merken sie, dass sie alle mit den gleichen Problemen kämpfen", sagt Professor Jean-François Chenot vom DEGAM-Präsidium. Zum Förderprogramm gehören die kostenlose
Teilnahme an den DEGAM-Kongressen und an der Summerschool für Allgemeinmedizin, die wissenschaftliche Fortbildung und Förderung und die Unterstützung bei Studium, Promotion und Berufsplanung, etwa durch ein Mentoring durch Allgemeinmediziner. „Wir sind dafür noch auf der Suche nach erfahrenen Ärzten", sagt Chenot. Geplant ist auch eine Famulaturbörse. Unterstützt wird die DEGAM dabei von der Gesellschaft der Hochschullehrer für Allgemeinmedizin und dem Deutschen Hausärzteverband.

Beim nächsten Treffen im Herbst auf dem Jahreskongress der DEGAM in Rostock ist die Zukunft der hausärztlichen Versorgung auf dem Land das Haupt-

INE = thema. 